FORMATION Formation emploi

Revue française de sciences sociales

101 | janvier-mars 2008

Numéro anniversaire : Regards croisés sur les

relations formation-emploi

\title{
Professionnaliser la formation : enjeux, modalités, difficultés
}

Professionalising vocational training: the challenges, modes and problems involved

Problematik der Professionalisierung der Bildung

Profesionalizar la formación: desafíos, modalidades, dificultades

\section{Richard Wittorski}

\section{(2) OpenEdition \\ Journals}

\section{Édition électronique}

URL : http://journals.openedition.org/formationemploi/1115

DOI : 10.4000/formationemploi. 1115

ISSN : 2107-0946

\section{Éditeur}

La Documentation française

\section{Édition imprimée}

Date de publication : 1 janvier 2008

Pagination : 105-117

ISSN : 0759-6340

Référence électronique

Richard Wittorski, «Professionnaliser la formation : enjeux, modalités, difficultés », Formation emploi [En ligne], 101 | janvier-mars 2008, mis en ligne le 31 mars 2010, consulté le 30 octobre 2020. URL: http://journals.openedition.org/formationemploi/1115; DOI : https://doi.org/10.4000/ formationemploi. 1115 


\title{
Numéro anniversaire
}

\section{Professionnaliser la formation : enjeux, modalités, difficultés}

\author{
Par Richard Wittorski ${ }^{*}$
}

\begin{abstract}
La professionnalisation est devenue un incontournable. Pourquoi souhaite-t-on, depuis quelques décennies, professionnaliser l'offre de formation initiale et continue? Avec quelles modalités? Quelles difficultés cela soulève-t-il ?
\end{abstract}

Comme le note Labruyère (2000), le couple «professionnalisation/compétence» s'impose de façon croissante dans les milieux du travail et de la formation. Quelle est l'offre de formation qui, aujourd'hui, ne se réclame pas d'une visée professionnalisante ? À la fois portée par les orientations nationales et européennes s'agissant de l'organisation de la formation initiale et continue et prônée par les secteurs privés, la professionnalisation a d'évidence «le vent en poupe ».

Notre projet consiste ici à mieux comprendre à la fois les raisons pour lesquelles on souhaite, depuis quelques décennies, professionnaliser davantage l'offre de formation initiale et continue (via le développement de l'alternance, des stages, la participation plus large de professionnels à l'élaboration, la mise en œuvre et l'évaluation des formations, via l'habilitation des formations par des instances mixtes et professionnelles, ...), mais aussi les modalités prises par les dispositifs mis en place, et la nature des difficultés à « faire vivre » l'intention de professionnalisation.

Conscients que le vocable professionnalisation est apparu d'abord dans d'autres champs de pratiques sociales que celui de la formation, nous commence- rons notre propos en précisant les sens du mot professionnalisation, au travers des usages sociaux qui en sont faits, révélant ainsi des enjeux parfois convergents, souvent contradictoires selon les acteurs « utilisateurs » du mot (promoteurs ou usagers des dispositifs).

\section{PROFESSIONNALISATION : USAGES, SIGNIFICATIONS ET ENJEUX SOCIAUX}

Cette première partie a donc pour objectif de montrer que le mot professionnalisation, au travers de ses

* Richard Wittorski est professeur des Universités en Sciences de l'éducation à l'IUFM (Institut de formation des maîtres) de l'université de Rouen et au laboratoire CIVIIC (Centre interdisciplinaire sur les valeurs, les idées, les identités et les compétences). Ses deux derniers ouvrages sont : La professionnalisation en actes et en questions lavec M. Sorell 2005, L'Harmattan et Professionnalisation et développement professionnel, 2007, L'Harmattan. 
usages sociaux (en dehors du champ de la formation), demeure fortement polysémique, traduisant ainsi des enjeux variés qu'il nous semble utile de repérer en premier lieu pour poser le contexte dans lequel le mouvement de professionnalisation de la formation s'insère, notamment un contexte marqué par des débats sociaux vifs s'agissant de la prescription et de la lisibilité de l'activité.

Il est probablement utile de distinguer les conditions historiques d'apparition d'une préoccupation de professionnalisation dans trois espaces habituellement articulés mais qu'il est nécessaire de disjoindre pour repérer la diversité des enjeux. Il s'agit d'une part, de l'espace politique et social; d'autre part, de l'espace des individus et des groupes sociaux (l'organisation des groupes sociaux en professions); enfin, de l'espace des organisations (l'environnement de l'offre de travail).

De ce point de vue, le mot profession apparaît à la fin $\mathrm{du} \mathrm{XIX}^{\mathrm{e}}$ siècle et au début du XXe siècle dans les pays anglo-saxons, à l'initiative de groupes sociaux qui cherchent à obtenir ou à accroître leur place sur un marché concurrentiel. Le mot professionnalisation, dans ce contexte, a eu pour première signification de traduire le processus de constitution d'une profession. Chez nous, il est, le plus souvent, porté par d'autres enjeux et apparaît plus récemment, souvent à l'initiative des organisations (en lien avec le mot compétence, à partir des années 1970-1980, pour ce qui concerne la France); il accompagne une recherche plus grande de «flexibilisation» des personnes (nous en reparlerons plus loin dans cette partie). Il s'inscrit, nous l'avons montré par ailleurs (Wittorski, 2007), dans un mouvement plus large consistant à valoriser dans le même temps une libéralisation du marché, une décentralisation politique (vers un citoyen « professionnel»), organisationnelle (vers un salarié professionnel) et sociale (vers un individu sommé d'être capable de produire sa propre vie par lui-même, de devenir l'« entrepreneur » de sa propre vie). Le vocable professionnalisation apparaît alors dans un contexte marqué par de nouvelles valeurs sociales telles la culture de l'autonomie, de l'efficacité, de la responsabilité, du « mouvement».

Sur les plans politique et social, il semble en effet qu'un mouvement d'ensemble révélant des enjeux sociaux convergents se développe, traduisant notamment la valorisation d'une décentralisation politique (donner le pouvoir au niveau local avec la territorialisation), sociale et organisationnelle (transférer la responsabilité de l'efficacité, de la gestion des changements et de l'évaluation du travail au niveau des personnes). Dans ce mouvement d'ensemble, et pour l'accompagner, apparaît aujourd'hui un discours marqué ayant pour intention la responsabilisation accrue des personnes en tant que citoyens. Ce discours est lié à une volonté de " professionnalisation plus grande de la société » qui a pour fonction explicite d'accompagner une mutation du fonctionnement des institutions politiques et administratives : passer ainsi d'un pilotage centralisé à un pilotage décentralisé permettant de gérer plus efficacement, au niveau local, les questions qui surviennent. Il est probable que cette volonté de professionnalisation de la société a pour intention implicite à la fois la « mise en mouvement $»$ des personnes permettant, au final, leur acceptation d'un nouveau mode de « gouvernance sociale» (en lien avec la décentralisation évoquée plus haut) et le retour d'un citoyen (« citoyen professionnel ») doté de valeurs communautaires et d'un certain sens renouvelé de la vie en société. Tout ceci n'est pas loin de signer une pensée libérale insistant sur l'efficacité individuelle au service d'un nouveau modèle de société.

Les enjeux pour les groupes professionnels se situent, quant à eux, clairement au niveau de la constitution des professions. Comme le note Paradeise (2003), le mot profession apparaît dans un contexte de marché libre où les acteurs économiques ressentent le besoin de développer une rhétorique concernant leur contribution au marché pour conquérir et accroître leur place. C'est probablement à ce niveau qu'il convient de situer l'apparition, dès le début du $\mathrm{XX}^{\mathrm{e}}$ siècle, du mot profession dans les pays anglo-saxons, associé d'ailleurs à l'image de la profession libérale. En France, il apparaît dans un contexte différent qui est caractérisé par un État hiérarchique (en France, traditionnellement, l'État est très présent dans l'organisation d'un certain nombre d'activités et donc d'emplois qui font l'objet d'un classement hiérarchique). Dès lors, la profession ne repose pas tant sur le modèle de la profession libérale mais davantage sur celui des corps d'État (par exemple, le corps des 
magistrats, tous formés par l'École nationale de la magistrature et responsables et garants de l'exercice de la fonction judiciaire). L'enjeu est donc ici la mise en reconnaissance de soi dans l'environnement à des fins de conquête d'une meilleure place dans une hiérarchie étatique.

Les enjeux que représente la professionnalisation pour les individus s'expriment sous la forme d'une quête de « professionalité » dans les organisations ou d'identité dans la sphère sociale.

Pour Dubar (2004, p. 141), «la crise des identités professionnelles s'inscrit dans une remise en cause générale des identités sociales, qui traduit le passage de relations communautaires (le nous) à des relations sociétaires (le je, au sens de la façon dont Weber caractérise le passage à la modernité). Produites autrefois collectivement, les identités professionnelles tendent désormais à être bricolées par les individus en fonction de leurs trajectoires professionnelles. Jusqu'au milieu des années 70, dans le cadre de relations communautaires, la qualification déterminait la production des identités collectives, à partir de systèmes stables de négociation d'équivalences entre employeurs et employés. Désormais, sous l'effet du chômage et de la libéralisation, dans des relations sociétaires, ce qui compte c'est le résultat que va apporter chaque individu à l'entreprise, accompagné du déclin des syndicats ». La quête de professionalité de la part des acteurs sociaux s'inscrit probablement dans cette recherche d'une nouvelle identité.

Les enjeux portés par les organisations (entreprises) ne concernent pas, selon nous, la constitution des professions dans l'espace social mais la professionnalisation des salariés entendue comme une intention organisationnelle d'accompagner la flexibilité du travail (modification continue des compétences en lien avec l'évolution des situations de travail). Ainsi, les caractéristiques des nouvelles organisations (production tirée par l'aval, intégration des activités et décloisonnement des fonctions) qui apparaissent au cours de ces trente dernières années génèrent trois conséquences : l'élargissement et l'enrichissement des compétences et des tâches, la réduction de la ligne hiérarchique, le développement du caractère collectif du travail. Comme le notent Berton, Boru et
Barbier (1996, p. 148), «ces transformations amènent à définir quatre nouvelles catégories de savoirs : des savoirs organisationnels » (construire la cohérence de l'amont/aval du poste), «des savoirs méthodologiques » (observer, classer...), « des méta savoirs» (conduite de processus d'action...), des «savoirs contingents au collectif de travail»" (l'ensemble des savoirs mobilisés dans un collectif de travail sans que ceux-ci soient également maîtrisés par chacun). Pour sa part, Coriat (1990, p. 169) parle d'une recomposition des figures ouvrières dans trois directions : "l'ouvrier fabricant (polyvalence et responsabilités), l'ouvrier technologue (tâches techniques habituellement réalisées par des techniciens) et l'ouvrier gestionnaire (gestion de paramètres économiques: coûts de production...)».

En lien avec ce qui précède, le discours des organisations sur la professionnalisation est récent; il fait ainsi écho à plusieurs évolutions fortement articulées entre elles :

- le passage d'une logique de production poussée par l'offre (l'entreprise planifie le travail) à une logique de production tirée par la demande (les salariés sont alors invités à être «acteurs et auteurs du changement »);

- le passage à une logique du résultat ;

- le passage d'un système contrôlé en son centre à une certaine décentralisation des responsabilités.

Le discours organisationnel sur la professionnalisation aurait donc notamment pour enjeu de susciter et de faire accepter ces évolutions.

Il semble bien, comme le notent plusieurs auteurs, que l'appel à la professionnalisation rejoigne, par ailleurs, un appel grandissant aux compétences mises au service des nouvelles normes de travail. Selon Dugué (1999, p. 14), les enjeux consistent à «faire avaler la pilule de la flexibilité ». Pour Stroobants (1993), l'adaptabilité permanente génère une individualisation de l'évaluation qui conduit, selon Linhart (1999, p. 59), à «resserrer le contrôle ». Pour cette même auteure (p. 62), il s'agit là de «stimuler les ressorts individuels». Le recours à la compétence traduit «un surcroît d'exigence vis-à-vis du salarié, celui d'avoir à s'organiser lui-même pour répondre aux insuffisances du travail prescrit, développer une 
nouvelle forme de performance centrée sur le service rendu aux clients et capable de mobiliser des ressources spécifiquement humaines de raisonnement et de décision» (Lichtenberger, 1999, p. 71). Nous sommes là dans le cadre d'un projet qui consiste à «mobiliser la subjectivité de tous» (Durand, 2000, p. 18). En quelque sorte, il s'agit d'une injonction pour que le sujet construise son expérience (Dubet, 1994). De ce point de vue, la professionnalisation présente une connotation positive au service d'une nouvelle mobilisation des salariés dans des contextes de travail plus flexibles, faisant davantage appel aux ressources subjectives des personnes.

$\mathrm{Au}$ total, le mot professionnalisation demeure fortement polysémique ; son usage est large mais les sens qui lui sont attribués sont multiples. Nous identifions ainsi au moins trois sens à l'œuvre dans les pratiques sociales :

- la professionnalisation-formation (sens utilisé par les formateurs: il s'agit de favoriser le développement des savoirs et des compétences) ;

- la professionnalisation-profession (sens utilisé par les acteurs sociaux : il s'agit de se constituer comme profession);

- la professionnalisation-travail (sens utilisé par les organisations : il s'agit de développer la «flexibilité » des personnes au travail et, au final, leur plus grande efficacité).

Qu'il s'agisse d'une initiative de l'organisation ou des individus, on comprend donc pourquoi le thème de la professionnalisation entre de plein pied dans un débat social duquel il ne peut être absent. D'une certaine façon, il présente, sous un visage différent, le débat faisant prévaloir, côté organisation, la logique compétence (proposée dans un souci d'accompagnement des évolutions du travail et, probablement aussi, pour répondre à un enjeu implicite de repositionnement du pouvoir dans les organisations au profit des directions d'entreprises) et, côté acteurs, la logique qualification (proposée et négociée dans un souci de mise en reconnaissance des professionalités à l'initiative des salariés, dans les organisations). En effet, quand elle est à l'initiative des organisations, la professionnalisation s'inscrit dans une logique de gestion de l'activité par les compétences, alors qu'elle relève d'une tentative de définition de repères collectifs permettant une mise en reconnaissance sociale d'une activité commune à un groupe social quand elle est à l'initiative des groupes professionnels.

Tenant compte des conditions d'apparition et d'usage social du mot professionnalisation analysées ici, demandons-nous, dans la partie suivante, comment est née une intention de professionnalisation dans le champ de la formation et interrogeons-nous sur les enjeux poursuivis.

\section{POURQUOI PROFESSIONNALISER LA FORMATION ?}

La vocation professionnelle de la formation n'est pas nouvelle. Ainsi, depuis ses origines, la formation des médecins, par exemple, relève d'une logique d'articulation forte avec les situations professionnelles. Il en va de même pour les formations universitaires de type DUT ou DESS (diplôme universitaire de technologie et diplôme d'études supérieures spécialisées) qui ont cette vocation affirmée depuis leur création, même si elles ont eu longtemps un statut de filières «à part» dans les universités se réclamant, dans l'ensemble, d'une logique de production et de diffusion des savoirs scientifiques et techniques. Nous assistons au contraire aujourd'hui (et c'est ici que se situe la nouveauté) à un mouvement de généralisation de la professionnalisation de la formation dans lequel la «norme» devient la visée professionnalisante de l'offre de formation (à l'image de la mission confiée par les derniers textes à l'Université française).

\section{Une intention de professionnalisation de la formation}

Plusieurs événements ont favorisé, dans l'histoire récente, le développement d'une préoccupation pour la professionnalisation de l'offre de formation.

D'une part, comme le note Verdier (2001), «les années 1985-1995 constituent, sans conteste, un tournant majeur pour le système français d'éducation et de formation, à la hauteur de celui qui avait 
marqué la première moitié des années 60 : forte croissance des effectifs de l'enseignement supérieur, création de diplômes professionnels et de filières de formation (à l'époque, la voie technologique; plus récemment, un vif développement de l'apprentissage). Bien évidemment, les contextes économiques et sociaux dans lesquels interviennent les réformes de l'éducation et de la formation contribuent à différencier ces deux "tournants": forte croissance économique, nombreuses créations d'emplois, intégration d'importants flux de migrants d'origines rurale et étrangère d'un côté, faible expansion économique, croissance du chômage et précarisation de l'emploi, exclusion du marché du travail et progression de la pauvreté, de l'autre». Bouder et Kirsch $(2007$, p. 4) indiquent ainsi que le baccalauréat professionnel, créé en 1985, marque en effet le début d'une nouvelle ingénierie de la formation, caractérisée par les notions de référentiel d'activités ${ }^{1}$ et de référentiel de compétences ${ }^{2}$ (son «pendant ») : «la procédure d'élaboration de ce diplôme stipule que la définition d'un référentiel des activités professionnelles est obligatoire avant toute définition de contenus de formation. On assiste ainsi à un renversement par rapport aux pratiques antérieures, très axées sur les contenus des programmes de formation et les procédures d'évaluation. Il correspond à la volonté de changer l'image du diplôme, d'en faire un contrat passé avec les partenaires sociaux qui va attester d'un certain nombre de compétences dont dispose le titulaire du diplôme.» Cette démarche s'est ensuite étendue à l'ensemble des certifications professionnelles.

S'agissant de l'enseignement supérieur français, les licences professionnelles sont instaurées en 1999 pour répondre aux besoins spécifiques de bassins ou d'entreprises locales. Au plan européen, la déclaration de Bologne, énoncée en 1999 et signée par trente-neuf pays européens, promeut d'une part un schéma d'organisation des études supérieures (système LMD), et d'autre part un schéma de validation des contenus permettant de capitaliser des

\footnotetext{
${ }^{1}$ Un référentiel d'activités décrit, en les organisant, les activités attendues à un poste de travail.

${ }^{2}$ Un référentiel de compétences mentionne les compétences nécessaires à la réalisation des activités liées à la tenue d'un poste de travail.
}

crédits dans des établissements différents. L'organisation des études supérieures se fait selon le schéma $3 / 5 / 8$, ces chiffres correspondant à trois niveaux de diplôme: le Bachelor (en France, licence pour les diplômes nationaux), après trois années d'études supérieures; le master, après deux années supplémentaires d'études supérieures, soit cinq ans au total; le doctorat (ou $\mathrm{PhD}$ ), après trois années supplémentaires, soit huit ans au total.

D'autre part, le Conseil européen de Lisbonne, en 2000 , constitue une autre étape significative, notamment à partir du projet de coopération renforcée pour l'éducation et la formation professionnelle intitulé «Processus de Bruges-Copenhague ». Celui-ci institue l'European Credit in Vocational Education and Training (ECVET), un système européen d'accumulation et de transfert d'unités capitalisables pour l'enseignement professionnel qui suppose la mise en place de normes définies en termes de compétences (ainsi que 1'European Qualifications Framework $(E Q F)$, un cadre européen de qualification destiné à permettre le positionnement européen des certifications nationales et à articuler les cadres nationaux de certification présents ou à venir).

Cette incitation et cette valorisation politique d'une professionnalisation accrue des formations se traduisent, dans les pratiques, à la fois par une diversification des spécialités enseignées, comme le notent Béduwé, Espinasse et Vincens (2007), et par une tendance à la conception de curricula à base d'approche par compétence (l'exemple significatif à cet égard étant celui du Québec : voir les travaux de Monchatre, 2007).

\section{Pour quels enjeux?}

Mais pourquoi chercher à professionnaliser davantage l'offre de formation? Probablement peut-on repérer plusieurs niveaux d'enjeux :

- d'une part, un enjeu (politique et social) de mise en correspondance plus forte des formations et des emplois. Répondre ainsi à la fois à la critique traditionnellement adressée à la formation (notamment initiale) selon laquelle elle ne préparerait pas suffisamment à l'insertion sociale et professionnelle et à la nécessité ressentie de penser des offres de formation plus adaptables en fonction de l'évolution continue 
des systèmes d'activité dans le champ du travail. Il s'agit là également d'un enjeu plus large de lutte contre le chômage et d'augmentation globale de l'« efficacité » des systèmes de formation (passant par une « flexibilité » plus grande) ;

- de façon corrélative, un enjeu de développement d'une nouvelle légitimité pour les systèmes de formation qui trouvent là l'opportunité de montrer l'utilité sociale et économique de la formation et de repositionner «stratégiquement » leur activité ;

- enfin, très probablement aussi un enjeu de décloisonnement des milieux de l'enseignement (notamment universitaires) et des milieux professionnels. Cette intention nous semble très présente dans les choix politiques qui ont été faits dans une période récente.

Reste que la professionnalisation croissante de l'offre de formation constitue, selon nous, à la fois une chance et un risque : une chance, d'une part, qui conduit les milieux de la formation à s'intéresser, pour la première fois, à la dimension formative de l'activité et à ne plus séparer (en lien avec une épistémè classique) espace et lieu de formation et espace et lieu de travail (mais les penser dans le cadre d'un continuum d'ensemble); un risque, d'autre part, qui consisterait à articuler étroitement (voire exclusivement) tout acte de formation à une intention de développement professionnel des individus, en laissant à l'individu la responsabilité de son développement personnel; c'est en effet le risque associé à une tendance générale à la finalisation plus grande de la formation par rapport aux situations de travail.

\section{DES LEVIERS POUR PROFESSIONNALISER L'OFFRE DE FORMATION SUPÉRIEURE ${ }^{3}$}

Analysons quelques exemples de formation supérieure professionnalisante: la formation supérieure professionnelle des enseignants et des travailleurs sociaux, l'introduction de la VAE et ses consé-

${ }^{3}$ Cette partie de l'article, ainsi que la suivante, sont très largement inspirées du chapitre d'ouvrage intitulé «Professionnalisation et offre de formation en enseignement supérieur » (Wittorski, 2008). quences et précisons, à chaque fois, les leviers utilisés pour professionnaliser.

\section{La formation professionnelle des enseignants en IUFM}

La formation proposée en Institut universitaire de formation des maitres (IUFM) aux enseignants stagiaires reçus aux concours du second degré (Agrégation ou $\mathrm{Capes}^{4}$ ) repose sur le principe de l'alternance : les stagiaires, placés dans des collèges ou lycées, prennent en charge une ou plusieurs classes sur une année complète pendant laquelle ils sont, par ailleurs, présents à l'IUFM chaque semaine pour une à deux journées de formation. La formation délivrée à l'IUFM est d'abord dite de nature « disciplinaire» (savoirs relatifs aux disciplines enseignées), mais également dite «générale » (savoirs dits «transversaux» ou d'ordre pédagogique). Elle utilise enfin, à des fins de professionnalisation, le levier de l'analyse des pratiques et du mémoire professionnel.

À partir de quelques travaux empiriques réalisés, il semble que l'analyse de pratiques constitue effectivement un outil privilégié de professionnalisation dans la mesure où elle assure une articulation étroite entre le travail et la formation (l'acte de travail devient objet et occasion de formation dès lors qu'il fait l'objet d'une analyse par son auteur). Elle permet à la fois un « retour sur l'action » et la transformation des actions habituellement mobilisées au travail en connaissances sur l'action, traduisant ainsi un apprentissage à partir de l'action qui participe de la construction de l'expérience des sujets. Nous avons particulièrement mis en évidence ces dynamiques dans une recherche récente concernant les modalités et les effets des dispositifs d'analyse des pratiques proposés aux enseignants (Wittorski, 2003). Dans l'activité professionnelle, les enseignants stagiaires développent des façons d'agir «sur le tas », dans l'immédiateté, dans l'instant de la situation (qui ont souvent un statut d'imprévu). Bien souvent d'ailleurs, ces pratiques sont des formes d'ajustement particulier des choix effectués en amont qui s'avèrent, dans l'instant de leur mise en œuvre, inadéquats et nécessiter une adaptation (le public

${ }^{4}$ Certificat d'aptitude au professorat de l'enseignement secondaire. 
n'étant pas, par exemple, dans les dispositions souhaitées ou prévues,...). Ces «trucs de métier» ont pour particularité d'être produits dans la situation et donc d'être efficaces dans l'instant, mais rarement reproductibles à l'identique dans d'autres situations, car ils sont adaptés à des contextes particuliers. Ce que permet précisément l'analyse de pratiques, c'est d'offrir un espace de mise en mots de ces pratiques spontanées qui, sans ce lieu de parole, resteraient souvent incorporées à l'action, c'est-à-dire non identifiées par leurs auteurs (voir la notion de compétence incorporée 5 développée par J. Leplat (1995). L'analyse de pratiques repose souvent sur une démarche de groupe consistant à réunir des personnes partageant les mêmes activités et qui sont incités à décrire, tour à tour, des pratiques professionnelles vécues (vis-à-vis desquelles elles éprouvent souvent une difficulté), celles-ci sont ensuite discutées et analysées en groupe. L'analyse de pratiques permet alors d'identifier les pratiques incorporées à l'action, mais aussi, par le travail d'échange collectif, d'en saisir les tendances communes, les principes qui les organisent, ces derniers étant utiles pour développer d'autres pratiques de retour en situation professionnelle. C'est ce que nous entendons par "principe organisateur des pratiques », des « règles d'action» (à validité individuelle ou collective) régissant les pratiques professionnelles et susceptibles d'en générer d'autres. Prenons quelques exemples extraits des discours des stagiaires qui nous semblent renvoyer à ces principes organisateurs des pratiques (ces extraits sont tirés de la recherche mentionnée ci-dessus (Wittorski, 2003)) :

- « il faut gérer ses émotions en classe » (ce principe est identifié par certains stagiaires après un travail d'analyse de leurs propres difficultés de gestion de leurs émotions en classe lors du dispositif d'analyse de pratiques) ;

- «il faut se mettre en scène» (ce principe est exprimé par certains stagiaires après avoir analysé des moments d'interaction avec la classe qui avaient posé problème).

\footnotetext{
5 J. Leplat définit une compétence incorporée comme «faisant corps avec les actions qui les expriment, $[. .$.$] elles sont facilement$ accessibles, difficilement verbalisables, peu coûteuses sur le plan de la charge mentale, difficilement dissociables, très liées au contexte » (Leplat, 1995, p. 102).
}

Autrement dit, ces «principes organisateurs des pratiques» ont le statut de «règles d'action» permettant de guider la mise en œuvre des pratiques à venir. Ils sont identifiés-déduits par les stagiaires à partir de l'analyse de leurs pratiques grâce à cette posture réflexive qu'ils développent. Ceci assure non seulement une transformation des pratiques en connaissances, mais également un apprentissage au sujet des règles guidant leurs actions.

Par ailleurs, et sur un autre plan, l'analyse des pratiques permet de repérer les nouveaux besoins de formation qui émergent en cours d'exercice alterné du métier; elle se présente alors comme un moyen utile pour réinterroger la formation de manière à l'articuler plus étroitement avec les situations professionnelles vécues.

Le mémoire professionnel, quant à lui, relève de l'exploration d'une question d'action vécue par les enseignants stagiaires au cours de leur stage. Il s'agit alors de l'approfondir (mieux comprendre les travaux susceptibles de l'éclairer) et de proposer des pistes utiles et transférables dans l'exercice du métier. De ce point de vue, les deux dispositifs mentionnés, analyse de pratiques et mémoire professionnel, semblent constituer des «leviers 》 privilégiés, aux yeux de l'institution, pour favoriser la professionnalisation des enseignants en formation.

\section{Des formations professionnelles continues de travailleurs sociaux}

Les dispositifs DHEPS (diplôme des hautes études et pratiques sociales) et DSTS (diplôme supérieur de travail social), par exemple, s'adressent en dominante à des publics salariés, donc déjà engagés dans une activité professionnelle. C'est probablement là une des différences importantes avec le premier exemple évoqué; les enseignants stagiaires étant, dans leur grande majorité, des étudiants sans expérience professionnelle antérieure.

Ces dispositifs utilisent, de pair avec des activités de formation plus classiques, explicitement le levier de la recherche-action professionnelle qui est sollicité à l'occasion de la réalisation du mémoire (DSTS et DHEPS). Le travail demandé consiste souvent à définir une question-problème rencontrée par le stagiaire dans son exercice professionnel (en lien 
avec sa pratique), à mener ensuite un travail théorique et empirique à son sujet en vue de mieux comprendre ses contours et à proposer, enfin, des réponses « éclairées » susceptibles d'être transférées dans l'espace professionnel (plus tard, s'il s'agit d'un mémoire).

Comme nous le faisions remarquer en 1997 (Wittorski, 1997), la recherche-action professionnelle peut constituer un outil utile pour professionnaliser ou « re-professionnaliser» des acteurs placés dans un parcours de mobilité professionnelle. Cela correspond à un enjeu réel, notamment pour les catégories professionnelles dont les contextes d'activités bougent et qui doivent rompre avec leurs pratiques habituelles pour produire de nouvelles façons de travailler. Ainsi, les travailleurs sociaux, les animateurs et les agents de formation sont de plus en plus sollicités pour apporter des réponses nouvelles et spécifiques à des situations inédites. La formation de ces professionnels ne se suffit probablement plus d'une transmission déductive de savoirs en pariant sur leur transfert différé en situation professionnelle. La transformation des modèles d'action par imitation (la logique de la « réplique du modèle professé ») a certainement ses limites lorsque les acteurs sont placés dans des situations professionnelles nouvelles. Il leur faut alors produire de nouvelles stratégies d'action. Cette capacité à la production de changement peut être développée si les acteurs ont élaboré de nouvelles aptitudes à la prise de recul, à l'analyse, ... De ce point de vue, l'engagement dans une démarche de formation appelée «rechercheaction » ou « recherche-action professionnelle » ancrée sur les pratiques professionnelles, est, probablement, un moyen permettant de produire ces nouvelles capacités. Cette recherche professionnelle constitue ici une nouvelle forme de recherche articulée à l'action, au service de l'apprentissage, à partir de celle-ci.

De façon provisoire, nous dirons qu'elle s'apparente à une démarche constructiviste: l'action, en se faisant, génère de nouveaux modèles d'action et l'analyse de cette action conduit l'individu à produire un cadre de référence analytique de l'action (et non seulement un cadre de référence pour l'action) qui laisse des traces sur la poursuite de l'engagement dans l'action. Il s'agit donc d'un processus itératif doublement constructiviste dans la mesure où il transforme conjointement l'action et son agent par la production d'outils mentaux nouveaux d'accompagnement et de gestion du changement.

La démarche de « recherche-action professionnelle » serait alors à différencier de l'acte professionnel et de l'acte de recherche (universitaire) comme le propose le tableau 1. Le point commun de l'acte professionnel, de l'acte de recherche et de la rechercheaction professionnelle est la constitution d'un cadre de représentation de l'action. Celui-ci n'a pour autant pas les mêmes fonctions: il permet d'organiser/diriger l'action au moment où elle se déroule (acte professionnel), il permet de lire et comprendre l'action observée (acte de recherche) et il permet conjointement d'analyser, de questionner et d'organiser l'action en cours (recherche-action professionnelle).

\section{L'introduction de la VAE dans les universités}

L'introduction de la validation des acquis d'expérience (VAE) dans les universités a contribué, plus ou moins significativement, non sans réserves et résistances dans un certain nombre de cas, à un mouvement de professionnalisation de l'offre de formation. Elle a suscité d'abord la réécriture des objectifs de formation en compétences de manière à penser des « équivalences » avec les expériences professionnelles des candidats à la VAE. Cette réécriture a parfois été également l'occasion de réorienter de façon significative les contenus ou modalités de formation choisis en lien avec les objectifs de compétences (et non plus seulement de savoirs) visés ; dans d'autres cas, les contenus et modalités de formation sont demeurés inchangés.

Prenons l'exemple du diplôme universitaire de formateur conseil, le DUFCO, de l'université Paris 5: la nécessité de penser une articulation avec la licence professionnelle et le master, mais aussi de prendre en compte des demandes de VAE croissantes, ont conduit à réécrire, en 2004, les objectifs de formation en termes de compétences et à proposer une architecture de formation intégrant une nouvelle modalité de formation consistant en la conduite d'un «projet tutoré » relevant du conseil en formation (depuis l'analyse de la demande jusqu'à la proposition d'une action). 
Tableau 1

Acte de recherche, acte professionnel et recherche-action professionnelle

\begin{tabular}{|c|c|c|c|}
\hline & Acte de recherche & Acte professionnel & $\begin{array}{c}\text { Recherche-action } \\
\text { professionnelle }\end{array}$ \\
\hline $\begin{array}{l}\text { Construction } \\
\text { d'un cadre pour lire, } \\
\text { agir sur ou penser } \\
\text { l'action }\end{array}$ & $\begin{array}{l}\text { Construction d'un cadre } \\
\text { théorique préalable } \\
\text { ou en cours de recueil } \\
\text { d'informations } \\
\text { II a une fonction } \\
\text { de lecture/analyse } \\
\text { de l'action }\end{array}$ & $\begin{array}{l}\text { Production d'un cadre } \\
\text { fonctionnel } \\
\text { (représentations } \\
\text { fonctionnelles) } \\
\text { accompagnant l'action } \\
\text { II a une fonction } \\
\text { d'organisation de } \\
\text { l'action }\end{array}$ & $\begin{array}{l}\text { Conceptualisation } \\
\text { de l'action en cours } \\
\text { de réalisation } \\
\text { Fonction double de } \\
\text { questionnement/ } \\
\text { analyse et } \\
\text { d'organisation } \\
\text { de l'action }\end{array}$ \\
\hline $\begin{array}{c}\text { Statut et position } \\
\text { de l'acteur } \\
\text { par rapport à l'action }\end{array}$ & $\begin{array}{l}\text { Action observée } \\
\text { Le chercheur est extérieur } \\
\text { à l'action }\end{array}$ & $\begin{array}{l}\text { Action en cours } \\
\text { de réalisation } \\
\text { Le professionnel est auteur } \\
\text { de l'action }\end{array}$ & $\begin{array}{l}\text { Engagement " réfléchi » } \\
\text { (« réflexif ») dans l'action } \\
\text { Le chercheur et } \\
\text { le professionnel s'impliquent } \\
\text { et prennent du recul } \\
\text { par rapport à l'action }\end{array}$ \\
\hline
\end{tabular}

Autrement dit, et de façon plus large probablement, la VAE nous semble être une occasion externe qui peut concourir à la professionnalisation plus forte de l'offre de formation, si les résistances sont levées.

Par ailleurs, et sur un autre plan, celui de la professionnalisation des personnes, même si la VAE ne constitue pas à proprement parler un dispositif de formation formelle professionnalisant ${ }^{6}$, le parcours de constitution du dossier semble produire sur le candidat des effets formateurs non négligeables qui induisent une réappropriation de son expérience et une «re-construction» de son identité professionnelle. Le levier utilisé ici est celui de l'écriture sur la pratique qui caractérise en dominante le processus de constitution du dossier de candidature à la validation. (cf. Magnier et Werthe, 2001).

\footnotetext{
${ }^{6}$ Nous faisons ici la différence entre la formation formelle au sens d'organisée et explicitement prévue dans un espace-temps particulier dédié aux apprentissages, et la formation informelle qui relève davantage des apprentissages réalisés au fil de l'activité sans que les situations n'aient été principalement organisées à cette fin.
}

Cette écriture engage le sujet à porter un regard rétrospectif sur sa pratique, à la mettre en mot et à en changer le statut: d'action, elle devient énoncé sur l'action. Ce nouvel énoncé s'accompagne d'une prise de conscience de modalités d'action auparavant non questionnées qui enrichissent donc les acquis d'expérience du sujet. L'écriture sur la pratique est alors l'occasion d'un apprentissage sur soi. La difficulté consiste ensuite à faire valider ces énoncés par un jury de manière à obtenir une reconnaissance de ces acquis d'expérience.

\section{L'alternance conjuguée au retour réflexif}

Ces exemples montrent que les leviers qui semblent particulièrement mobilisés pour accompagner ou susciter la professionnalisation des individus dans les formations supérieures relèvent :

- d'une part, de l'alternance: de ce point de vue, pas de formation dite professionnalisante sans un temps plus ou moins long d'exercice professionnel 
en alternance avec un parcours de formation dans lequel l'action professionnelle nouvelle en cours de construction est, elle-même, réinterrogée ;

- d'autre part, d'une analyse orale ou écrite des pratiques professionnelles; il s'agit tantôt de groupes d'analyse de pratiques, tantôt de travaux d'écriture sur la pratique (mémoire professionnel, rechercheaction, ...).

Ces deux leviers sont, bien entendu, souvent fortement articulés dans les offres de formation. À côté des savoirs théoriques, ils permettent de « travailler » des savoirs non théoriques que l'on appelle souvent des « savoirs d'action » (Barbier et Galatanu, 2004) ou encore des savoirs professionnels. Par exemple, le fait de réinterroger, en formation, l'action professionnelle mise en œuvre in situ permet de changer le statut de l'action pour son auteur : ainsi devient-elle pour lui un acquis « su » de l'expérience, alors même que l'action considérée pouvait être fortement incorporée à la situation (et donc non identifiée par le sujet). Par ailleurs, cette mise en mots de l'action permet un apprentissage non seulement par le sujetauteur de l'action mais également par les participants partageant le même espace-temps de retour sur les pratiques (les uns apprennent de l'écoute des autres).

\section{LA DIFFICILE PROFESSIONNALISATION DE L'OFFRE DE FORMATION}

Prenons l'exemple de la licence professionnelle instaurée en 1999 en France. Une étude réalisée par le Céreq, entre 2001 et 2003, sur la professionnalisation de l'Université (Maillard et Veneau, 2003), montre que trois grands principes sont à l'œuvre : l'innovation pédagogique, la mixité des publics et le partenariat avec les milieux professionnels. Comme le disent ces auteurs (p. 1), «des principes qui ont parfois quelques difficultés à entrer dans les faits. Dans leur grande majorité, les licences professionnelles sont en effet mises en place à l'initiative des enseignants, les représentants du monde professionnel y jouent le plus souvent un rôle secondaire et elles accueillent essentiellement des étudiants titulaires d'un DUT ou d'un BTS. Si la création de la licence professionnelle n'a pas eu tous les effets escomptés, elle a néanmoins permis d'introduire de nouveaux cadres de mise en ouvre des formations professionnelles, notamment dans les UFR (unités de formation et de recherche) ». Un dispositif nouveau d'habilitation a été institué à l'occasion de la création des licences professionnelles : les projets de création de licences sont évalués par une commission nationale d'expertise composée de manière tri-partite, au sein de laquelle les représentants des organisations professionnelles d'employeurs et des syndicats de salariés ont une place équivalente à celle des représentants universitaires. Cela conduit à réduire la place qu'occupait auparavant le milieu universitaire dans le processus d'expertise des projets de formation (il en est de même du système des CPC commissions professionnelles consultatives - dans le secondaire ou des CPN - commissions pédagogiques nationales - pour les IUT - instituts universitaires de technologie...).

Maillard et Veneau (2003, p. 2) font, par ailleurs, le constat que «les licences professionnelles sont le plus souvent créées dans une "logique d'offre". Les enseignants sont à l'origine des projets, ont l'initiative des démarches et maîtrisent la rédaction des contenus. Ils formulent leur projet sur la base d'intuitions, d'idées, d'analyses plus ou moins détaillées de l'offre de formation et du marché de l'emploi, de contacts ou de relations établies à la suite de contrats de recherches, de stages étudiants, de formations déjà existantes comme par exemple les DU (diplômes d'université). Les modalités d'élaboration des projets, qui sont autant de manières de nouer des relations avec les milieux professionnels, prennent donc des formes diverses et sont d'intensité très variable. Il n'en demeure pas moins que l'implication des professionnels se réalise toujours dans un second temps, sur le mode de la réaction après sollicitation des enseignants ». Par ailleurs, les analyses montrent que les salariés d'entreprises restent peu présents dans les équipes d'enseignants.

Complémentairement, les difficultés parfois rencontrées dans les dispositifs à visée professionnalisante concernent les conditions effectives de l'articulation entre deux espaces traditionnellement disjoints : celui de la formation et celui du travail. Ces difficultés sont souvent reliées au transfert des acquis du 
premier espace vers le second ou à l'articulation des savoirs théoriques acquis dans le premier avec les savoirs d'action développés dans le second (voir les nombreux travaux sur l'alternance depuis 30 ans, notamment l'article de Benjamin Dubrion, 2006).

Nous pensons que ces difficultés sont plus fondamentalement dues à l'existence de cultures dominantes de pensée encore très présentes concernant la formation. Celles-ci renvoient à une épistémè classique qui a montré longtemps sa fonction sociale, conduisant souvent à compartimenter les deux espaces :

- une conception encore dominante selon laquelle le lieu professionnel est un lieu d'application des savoirs issus de la formation. Or, la réalité est bien différente puisque de nombreux travaux (tels ceux de Carré et Charbonnier (2003) qui parlent d'apprentissages professionnels informels) insistent sur le fait que les situations de travail sont également formatrices. Par ailleurs, il est montré que les savoirs issus de la formation ne se transfèrent pas automatiquement dans les pratiques ;

- cette conception a souvent pour corollaire l'idée que le savoir théorique est préalable à toute action professionnelle efficace et tend donc à "sacraliser» le savoir théorique et à attribuer à l'organisme de formation un rôle de «prescripteur» et au terrain professionnel un rôle d'« utilisateur». Or, comme le montrent certains travaux (notamment Schön, 1996 ou Tardif et Lessard, 2000) depuis une vingtaine d'années, l'action professionnelle recèle des savoirs tout aussi efficaces; parfois plus que ceux issus de l'activité scientifique. Ce qui explique d'ailleurs l'enjeu actuel relatif aux dispositifs d'analyse de pratiques qui se développent dans de nombreux secteurs en vue de repérer les pratiques efficaces et identifier des savoirs spécifiques au champ professionnel;

- cette conception s'accompagne enfin souvent d'une conception « élitiste » des savoirs, considérant la pratique comme de seconde valeur.

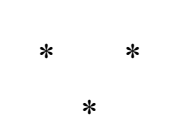

Pour conclure, nous aimerions insister sur les conditions que doit remplir, à nos yeux, une formation pour être professionnalisante. Une telle formation suppose probablement une analyse préalable du travail réel pour identifier les compétences à développer en formation (nécessité d'identifier des compétences visées et des compétences validées par le secteur professionnel). Elle suppose également de penser la formation par finalité d'action; or, on pense souvent spontanément la formation par apport disciplinaire. Elle suppose ensuite une diversification des modalités pédagogiques : au-delà du cours (les compétences ne s'enseignent pas, loin de constituer des énoncés, elles relèvent surtout de modalités d'action en situation), prévoir des mises en situation (propices au développement des compétences) et des moments d'analyse de pratiques (pour leur exploitation). Elle nécessite enfin de mettre en place une évaluation des compétences (c'est-à-dire une évaluation des modalités d'action effectives des sujets) et non seulement une évaluation des savoirs (c'est-à-dire des énoncés "déclaratifs 》 sur des propriétés d'objets ou d'actions). Cela conduit à partager le pouvoir de conception de la formation avec les milieux professionnels, tout en faisant évoluer le métier de formateur: un formateur capable d'élaborer des situations problèmes, de développer l'analyse de pratiques...

Bien plus, une formation professionnalisante nous semble devoir s'appuyer sur une conception « intégrative ou itérative» de l'alternance et des rapports travail-formation en lien avec un nouveau paradigme (qui reste à construire) susceptible de penser la continuité et la complémentarité entre les deux espaces du travail et de la formation. Les sujets sont alors invités à développer une posture réflexive rétrospective par rapport à leur action (modèle du praticien réflexif) permettant de construire l'expérience « sue ». L'analyse de pratiques articule alors les deux espaces du travail et de la formation. On parle non seulement de savoirs théoriques mais aussi de savoirs d'action. Mais cette conception entre en contradiction avec une conception «applicative ou déductive » de l'alternance encore très vivace, nous l'avons noté plus haut, qui a tendance à séparer travail et formation et à consacrer la suprématie de la théorie sur la pratique: la logique de l'offre de formation formelle prédomine. 
Barbier J.-M. et Galatanu O. (Éds.) (2004). Les savoirs d'action : une mise en mot des compétences? Paris, L'Harmattan.

Béduwé C., Espinasse J.-M. et Vincens J. (2007), « De la formation professionnelle à la professionnalité d'une formation ». Formation Emploi, 99, juilletseptembre 2007, pp. 103-121.

Berton F., Boru J.-J. et Barbier J.-M. (1996), Situations de travail et formation, Paris, L'Harmattan.

Bouder A. et Kirsch J.-L. (2007), « La construction de l'Europe de la compétence », Bref-Céreq, 244, 4 p.

Carré P. et Charbonnier O. (2003), Les apprentissages professionnels informels, Paris, L'Harmattan.

Coriat B. (1990), L'atelier et le robot, Paris, Bourgeois.

Dubar C. (2004), «Identités professionnelles : le temps du bricolage », in Identité(s) : l'individu, le groupe, la société Paris, Sciences Humaines, pp. 141-148.

Dubet F. (1994), Sociologie de l'expérience, Paris, Seuil.

Dubrion B. (2006), «Qu'est-ce qu'un bon apprenti du supérieur? », Formation Emploi, 96, pp. 93-105.

Dugué E. (1999), « La logique compétence : le retour du passé », Éducation Permanente, 140, pp. 7-18.

Durand J.-P. (2000), «Les enjeux de la logique compétence », Annales des mines, 62, pp. 16-24.

Labruyère C. (2000) « Professionnalisation : de quoi parlent les chercheurs, que cherchent les acteurs?» Formation Emploi, 70, pp. 31-42.

Leplat J. (1995), « À propos des compétences incorporées », Éducation Permanente, 123, pp. 101-114.
Lichtenberger Y. (1999), « La compétence comme prise de responsabilité », in Club CRIN (Éd.) Entreprises et compétences : le sens des évolutions, Paris, Les cahiers du club CRIN, pp. 69-85.

Linhart D. (1999), « Des entreprises modernisées, des salariés désarmés », Éducation Permanente, 141, pp. 55-67.

Magnier J. et Werthe Ch. (2001), «L'expérience revisitée à l'occasion de la VAP », Formation Emploi $\mathrm{n}^{\circ} 75$, pp. 29-41.

Maillard D. et Veneau P. (2003), «Les licences professionnelles », Bref-Céreq, 197, 4 p.

Monchatre S. (2007), «En quoi la compétence devient-elle une technologie sociale ? Réflexions à partir de l'expérience québécoise », Formation Emploi, 99, pp. 29-45.

Paradeise C. (2003), « Comprendre les professions : l'apport de la sociologie », Sciences Humaines, 139, pp. 26-29.

Schön D.A. (1996), « À la recherche d'une nouvelle épistémologie de la pratique et de ce qu'elle implique pour l'éducation des adultes », in J.-M. Barbier (Éd.) Savoirs théoriques et savoirs d'action, Paris, PUF, pp. 201-222.

Stroobants M. (1993), Savoir-faire et compétences au travail, Éditions de l'université de Bruxelles.

Tardif M. et Lessard C. (2000), Le travail enseignant au quotidien, Louvain, De Boeck.

Verdier É. (2001), « La France a-t-elle changé de régime d'éducation et de formation?» Formation Emploi, 76, pp. 11-34.

Wittorski R. (1997), Analyse du travail et production de compétences collectives, Paris, L'harmattan.

Wittorski R. (2003) (dir.), Analyse de pratiques et professionnalisation des enseignants, CNAM, Centre de Recherche sur la Formation. 
Wittorski R. (2005) (dir.), Travail, formation et professionnalisation, Paris, L'harmattan.

Wittorski R. (2007), Professionnalisation et développement professionnel, Paris, L'Harmattan.
Wittorski R. (2008), « Professionnalisation et offre de formation en enseignement supérieur ", in Hébrard $\mathrm{P}$. et Solar C. (2008) (Éds.), Professionnalisation et formation des adultes: une perspective universitaire France-Québec, Paris, L’Harmattan, pp. 47-70.

\section{Résumé}

\section{Professionnaliser la formation: enjeux, modalités, difficultés}

Quelle est l'offre de formation qui, aujourd'hui, ne se réclame pas d'une visée professionnalisante ? À la fois portée par les orientations nationales et européennes s'agissant de l'organisation de la formation initiale et continue et prônée par les secteurs privés, la professionnalisation a d'évidence "le vent en poupe ».

Notre projet consiste ici à mieux comprendre à la fois les raisons pour lesquelles on souhaite, depuis quelques décennies, professionnaliser l'offre de formation initiale et continue, les modalités prises par les dispositifs mis en place, et la nature des difficultés à « faire vivre »l'intention de professionnalisation.

\section{Mots clés}

Marché de la formation professionnelle, système éducatif, professionnalisation, enseignement universitaire, validation des acquis

Journal of Economic Literature: J 44 - Professional Labor Markets ; I 21 - Analysis of Education 
